\title{
Synchrotron based X-ray fluorescence for trace elemental analysis of industrial sludge
}

\author{
Vijay Kumar Garg ${ }^{1}$, Arun Lal Srivastav², Manoj Kumar Tiwari², Ajay Sharma ${ }^{1 *}$ and \\ Varinder Singh Kanwar ${ }^{1}$
}

\begin{abstract}
${ }^{1}$ Chitkara University School of Engineering and Technology, Chitkara University, Himachal Pradesh-174103, India
2 BL-16, Indus-2, Raja Ramana Centre for Advanced Technology, Indore, India
\end{abstract}

Received: 12/08/2020 Accepted: 01/11/2020_ Published: 20/03/2021

\begin{abstract}
This research article presents the application of the synchrotron-based X-ray fluorescence (XRF) technique for the resolution of trace elemental accumulation in industrial sludge/waste. The X-ray fluorescence using synchrotron radiation presents an expeditious exposition of a wide scale of elements (Sodium to Uranium) together with an ingenuous sample preparation procedure. The present Xray fluorescence studies carried out for the paper and toothpaste industry sludge at synchrotron source (Beam Line -16), Indus-2, Raja Ramanna Centre for Advanced Technology, Indore, India. The XRF results show very low traces of heavy metals present in paper and toothpaste industry sludge and therefore recommend for safe and efficient reuse.
\end{abstract}

Keywords: Elemental analysis; synchrotron radiation; industrial sludge and X-ray fluorescence

\section{Introduction}

In developing countries, industrial sectors pose significant environmental and occupational health risks to its populations. As industries are booming and large quantities of industrial waste and are being dumped illegally either in open or in the nearby riverbeds. World's urban societies are increasing rapidly than the global population, especially in the developing countries. Urban growth has created momentous alteration to the ecological system by increasing waste accumulation through human activities. The techniques which are capable to detect the level of metallic elements to ppb limits in a variety of environmental, biological as well as geological samples are;

- Atomic absorption spectroscopy (AAS)

- Inductively coupled plasma-atomic emission spectrometry (ICP-AES) or inductively coupled plasma-mass spectrometry (ICP-MS) or Inductively coupled plasmaoptical emission spectrometry (ICP-OES).

- Energy-dispersive X-ray Fluorescence Spectrometry (EDXRF) or Wavelength dispersive XRF (WD-XRF)

- Neutron activation analysis (NAA)

- Particle-induced X-ray emission (PIXE)

For the last 20 years, the energy dispersive X-ray fluorescence (EDXRF) has advanced diligently. The developments made in semiconductors, digital signal processing and data simulation have broadened the usability of the XRF method for economical, small-size spectrometers for trace elemental analysis in various types of samples [1-3]. X-ray fluorescence being a non-destructive analytical method has its own merits. XRF analysis provides the composition of a sample by quantifying the characteristic X-rays evolved from a sample, excited by radiations (Fig. 1). In X-ray fluorescence, individual atoms are excited by incident photons, which in turn emit secondary photons called as characteristic X-rays (Fig. 1a). In XRF spectroscopy, emission of characteristic X-rays of the sample is atomized because of the primary incident X-ray or gamma ray photons. The energy and intensity of the characteristic X-rays in the XRF spectra indicate the element present and its concentration in the sample. XRF is an important technique of elemental analysis for distinct kind of samples and it is an extremely responsive technique, which follows the principle of interaction of atoms with radiation [46]. The principal asset of the X-ray fluorescence method over other elemental analysis techniques is its non-destructiveness, simultaneous multi-elemental capability, ingenuous sample preparation and high resolution for trace elements. Daly et al. [7] used EDXRF for elemental analysis dairy processing sludge before its application in the agricultural fields and concluded that the XRF method is provides rapid and accurate results. Mashaly et al. [8] characterized the granite sludge and cement on the basis of X-ray fluorescence and studied the feasibility of granite sludge in cement replacement. Elemental composition of solid residues of sewerage sludge and biomass waste were examined using XRF [9]. Zhang et al. [10] evaluated the textile dyeing sludge and cattle manure using XRF and their findings provided an insight for better reutilization of waste. Synchrotron-based XRF can be used to know both quantitative and qualitative multi-elemental concentration in very less time [11] and it is a non-destructive, most precise and accurate method of trace element determination of all types of samples. Furthermore, it can simultaneously determine many elements present in a single sample [12]. In India, this facility is available at Raja Ramanna Centre for Advanced Technology (RRCAT), Indore. Presently this technique is being used for the trace elemental analysis of sludge samples collected from the paper and toothpaste industry, which is abundant in the study area.

*Corresponding author: Ajay Sharma, Chitkara University School of Engineering and Technology, Chitkara University, Himachal Pradesh-174103, India. E-mail: coe@ chitkarauniversity.edu.in 


\section{Material and method}

Presently, paper and toothpaste industry sludge samples are gathered from an industrial area. The collected powder samples are pelletized by uniformly mixing with cellulose to make $13 \mathrm{~mm}$ diameter self-supporting targets with a smooth surface of equal density (Fig. 2). These targets are evaluated at the synchrotron radiation beam line-16 at RRCAT, which is specially designed and commissioned by Department of Atomic Energy (DAE) for XRF/TXRF (Fig. 3a). Owing to the numerous advantages of a synchrotron-based XRF technique the experimental set-up on the Indus-2 synchrotron light source is equipped with a microfocus XRF beamline (BL-16). The beamline (BL-16) is capable of radiating monochromatic, intense radiations in the energy span 4 to $20 \mathrm{keV}$. At the experimental hutch, the beamline presents both micro-focused and collimated beam modes for versatile applications. The energy dispersive X-ray fluorescence (EDXRF) and total reflection $\mathrm{X}$-ray fluorescence (TXRF) analysis of samples up to $\mathrm{ppb}$ (parts per billion) levels can be made by exposing the synchrotron beam for a short duration of time. In the present measurements, the sludge pellet samples were exposed to BL16 at $18 \mathrm{keV}$ energy. Sludge pellets with thickness of 0.2003 $\mathrm{gm} / \mathrm{cm}^{2}$ were prepared for experimentation purposes. The experimental set-up (Fig. 3b) consists of a sample holder inclined at $45^{\circ}$ to the incident radiations in the reclining divergence arrangement. The characteristic X-rays emitted from the sludge samples were detected by a Peltier cooled Vortex solid-state Silicon drift detector (SII Nano, USA) for 500 seconds. The detector with an energy resolution of $138 \mathrm{eV}$ at $5.9 \mathrm{keV}(\mathrm{Mn} \mathrm{K} \alpha) \mathrm{X}$-rays was mounted at $90^{\circ}$ to the incident beam. The detector was lying approximately $60 \mathrm{~mm}$ away from the sample surface (middle of the beam footmark). To escalate the flux and to focus the beam size a double crystal monochromator and a 200-micrometer slit was pressed into the hutch, the same helped in optimizing beam strength and mitigating the background scattering.

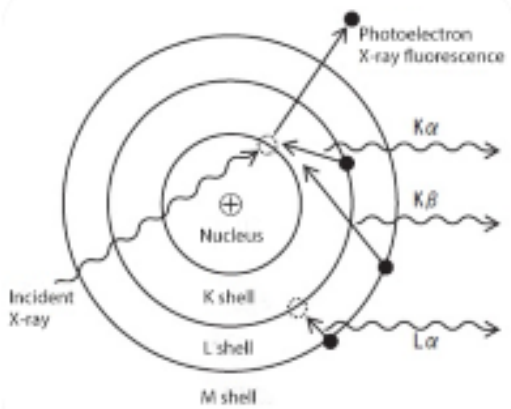

(a)

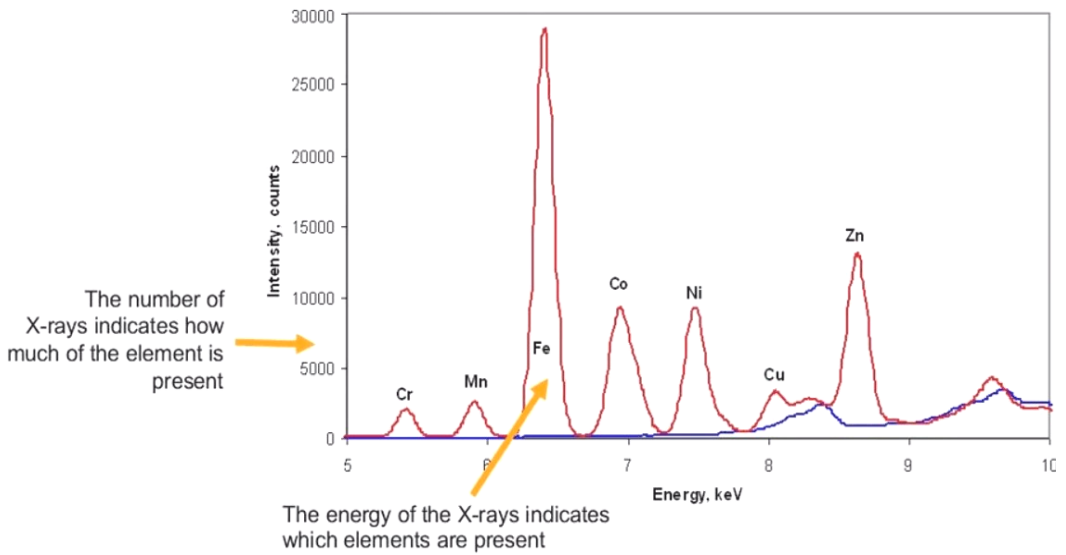

(b)

Fig. 1 (a) The mechanism of X-ray fluorescence and (b) the typical XRF spectrum
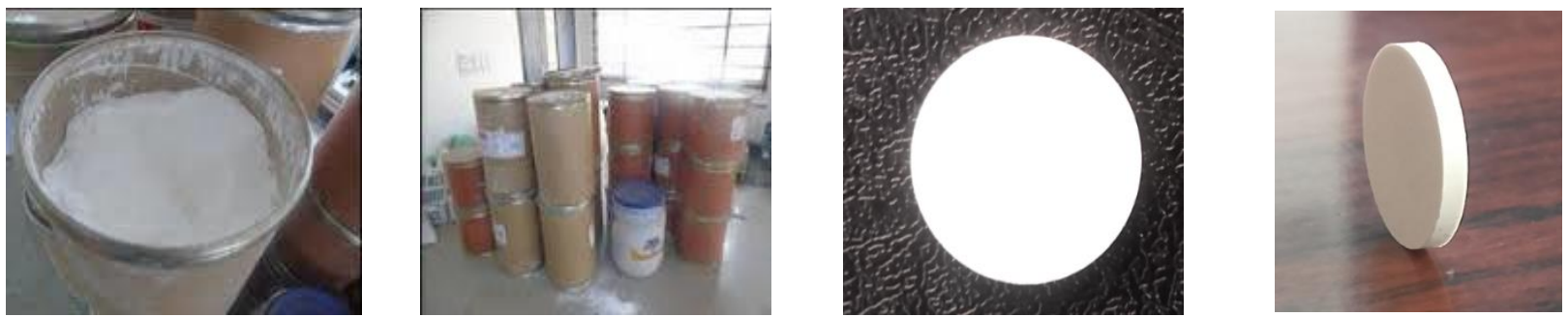

Fig. 2: Sludge powder and Pellet samples for XRF study

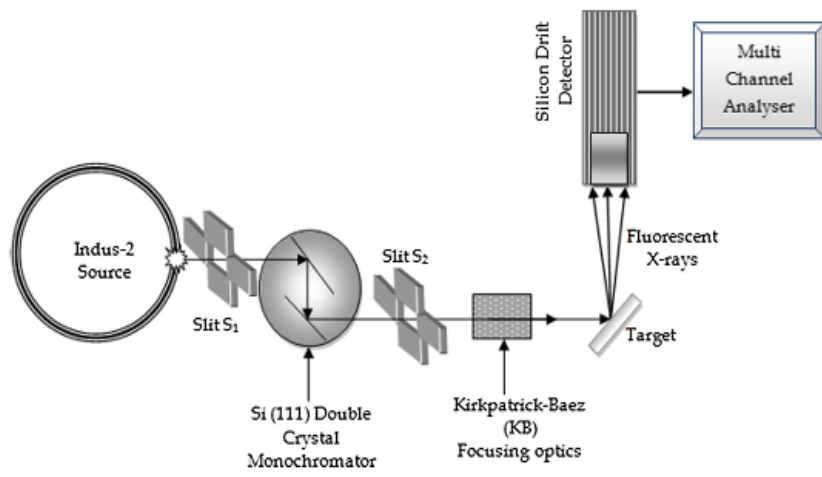

(a)

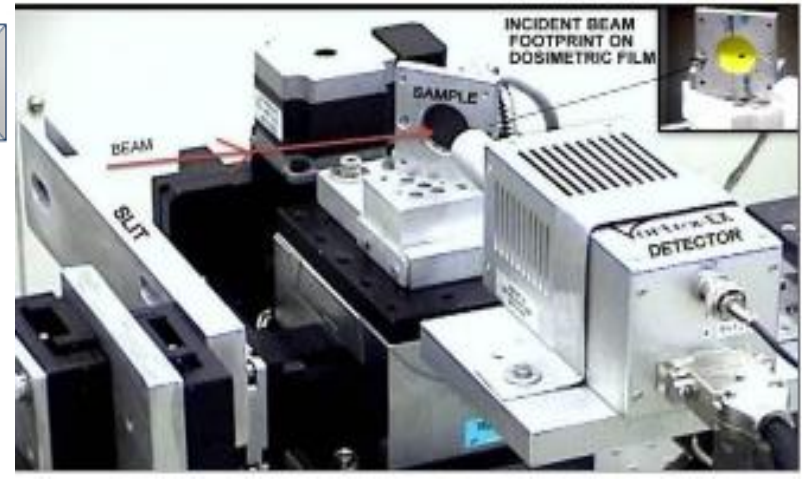

(b)

Fig. 3: (a) Schematic layout of the experimental set-up (b) Actual photograph of the hutch 


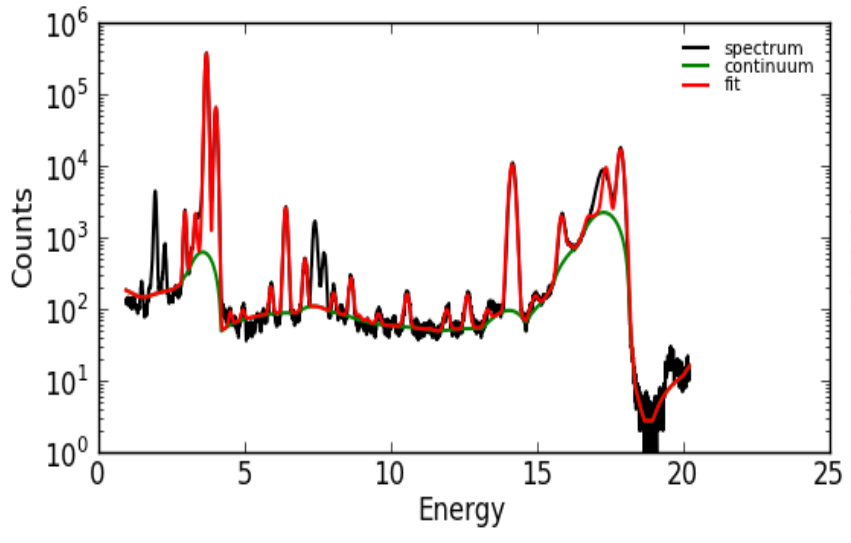

(a)

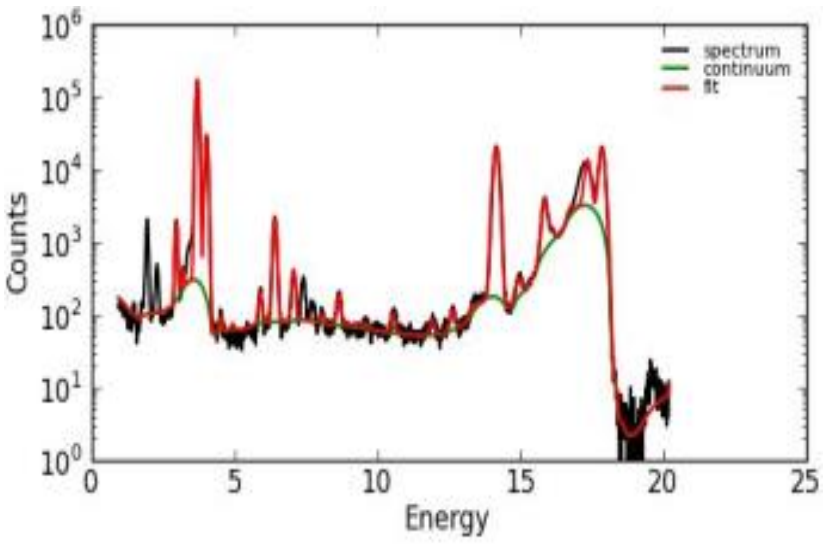

(b)

Fig. 4: Fitted fluorescent characteristic X-ray spectra for (a) paper sludge (b) toothpaste sludge

Table 1: Elemental concentration (in ppm) for paper sludge and toothpaste sludge alongwith $7 \%$ experimental uncertainty

\begin{tabular}{llcc}
\hline Sr. No. & Element & Paper sludge $(\mathrm{ppm})$ & Toothpaste sludge $(\mathrm{ppm})$ \\
\hline 1 & $\mathrm{Ca}$ & $937683( \pm 65638)$ & $801358( \pm 56095)$ \\
2 & $\mathrm{Ti}$ & $88( \pm 6)$ & $181( \pm 13)$ \\
3 & $\mathrm{Mn}$ & $374( \pm 26)$ & $763( \pm 53)$ \\
4 & $\mathrm{Fe}$ & $8063( \pm 564)$ & $12089( \pm 846)$ \\
5 & $\mathrm{Cu}$ & $280( \pm 20)$ & $168( \pm 12)$ \\
6 & $\mathrm{Zn}$ & $724( \pm 51)$ & $810( \pm 57)$ \\
7 & $\mathrm{Br}$ & $217( \pm 15)$ & $260( \pm 18)$ \\
8 & $\mathrm{Sr}$ & $51827( \pm 3628)$ & $182591( \pm 12781)$ \\
9 & $\mathrm{Y}$ & $270( \pm 19)$ & $1396( \pm 98)$ \\
10 & $\mathrm{~Pb}$ & $47( \pm 3)$ & $23( \pm 2)$ \\
\hline
\end{tabular}

\section{Results and discussion}

Typically recorded characteristic X-ray spectra at $18 \mathrm{keV}$ is shown in Fig. 4. In order to control the characteristic parameters of the beam line such as spectral range and minimum detection limit etc. the sludge samples have been exposed to radiations for 500 seconds. Fig. 4 shows fitted XRF spectra, where solid black and red lines are the experimental and fitted data respectively, whereas green line represents a good estimation of the spectral background.

The XRF results (table 1) show the dominating proportion of Calcium in both sludge samples. The concentration of titanium, manganese, iron and zinc is more in toothpaste sludge as compared to paper sludge, whereas the concentration dominates for copper and lead in paper industry sludge. The comparative high concentration of titanium, manganese in toothpaste sludge may be due to the small percentage of their compounds as an ingredient in the toothpaste. The present results (table 1) show very low traces of heavy metals and a dominating concentration of calcium in both the sludge samples, which recommend these industrial sludge(s) for safe and efficient recycling. Similar findings have already been reported for elemental analysis dairy processing sludge before its application in the agricultural fields using EDXRF [7]. Therefore, XRF based multi-elemental, qualitative and quantitative valuable information can be considered while recommending any sludge for reuse. The researchers are also working on the techniques of safe disposal of these wastes through various techniques like phytoremediation [13].

\section{Conclusion}

As is evident from the present study, synchrotron-based Xray fluorescence is a significant technique for non-destructive multielemental analysis of industrial waste/sludge. The technique exhibits both qualitative and quantitative information of trace elements present in sample in precise and accurate manner. The present XRF results predict the dominating concentration of Calcium in industrial sludge/waste and therefore, can be an alternate in replacing soil in brick manufacturing industry.

\section{Acknowledgment}

The authors would like to thank the Department of Environment, Science \& Technology, Himachal Pradesh-India for financial support in this study vide grant no. Env. S \& T (F)/5-1/2018-8886. The authors also acknowledge the technical assistance and guidance extended by RRCAT Indore-India to carry on this study.

\section{Ethical issue}

Authors are aware of, and comply with, best practice in publication ethics specifically with regard to authorship (avoidance of guest authorship), dual submission, manipulation of figures, competing interests and compliance with policies on research ethics. Authors adhere to publication requirements that submitted work is original and has not been published elsewhere in any language.

\section{Competing interests}

The authors declare that there is no conflict of interest that would prejudice the impartiality of this scientific work.

\section{Authors' contribution}

All authors of this study have a complete contribution for data collection, data analyses and manuscript writing. 


\section{References}

1. Bamford S, Wegrzynek D, Chinea-Cano E, Markowicz A. Application of X-ray fluorescence techniques for the determination of hazardous and essential trace elements in environmental and biological materials. Nukleonika. 2004;49(3):87-95.

2. Schramm R, Heckel J. Fast analysis of traces and major elements with ED (P) XRF using polarized X-rays: TURBOQUANT. Le Journal de Physique IV. 1998 Oct 1;8(PR5):Pr5-335.

3. Chen L, Liao Y, Ma X, Niu Y. Effect of co-combusted sludge in waste incinerator on heavy metals chemical speciation and environmental risk of horizontal flue ash. Waste Management. 2020 Feb 1;102:645-54.

4. Török SB, Lábár J, Schmeling M, Van Grieken RE. X-ray spectrometry. Analytical Chemistry. 1998 Jun 15;70(12):495-518.

5. Anjos MJ, Lopes RT, Jesus EF, Assis JT, Cesareo R, Barroso RC, Barradas CA. Elemental concentration analysis in soil contaminated with recyclable urban garbage by tube-excited energy-dispersive X-ray fluorescence. Radiation Physics and Chemistry. 2002 Nov 1;65(4-5):495-500.

6. Soodan RK, Pakade YB, Nagpal A, Katnoria JK. Analytical techniques for estimation of heavy metals in soil ecosystem: a tabulated review. Talanta. 2014 Jul 1;125:405-10.

7. Daly K, Fenton O, Ashekuzzaman SM, Fenelon A Characterisation of dairy processing sludge using energy dispersive X-ray fluorescence spectroscopy. Process Safety and Environmental Protection. 2019 Jul 1;127:206-10.

8. Mashaly AO, Shalaby BN, Rashwan MA. Performance of mortar and concrete incorporating granite sludge as cement replacement. Construction and Building Materials. 2018 Apr 30;169:800-18.

9. Chen J, He Y, Liu J, Liu C, Xie W, Kuo J, Zhang X, Li S, Liang J, Sun S, Buyukada M. The mixture of sewage sludge and biomass waste as solid biofuels: Process characteristic and environmental implication. Renewable Energy. 2019 Aug 1;139:707-17.

10. Zhang J, Sun G, Liu J, Evrendilek F, Buyukada M. Co-combustion of textile dyeing sludge with cattle manure: Assessment of thermal behavior, gaseous products, and ash characteristics. Journal of Cleaner Production. 2020 Apr 20;253:119950.

11. Ene A, Bosneaga A, Georgescu L. Determination of heavy metals in soils using XRF technique. Rom. Journ. Phys. 2010 Jan 1;55(78):815-20.

12. Radtke M, Reinholz U, Gebhard R. Synchrotron RadiationInduced X-Ray Fluorescence (SRXRF) Analyses Of The Bernstorf Gold. Archaeometry. 2017 Oct;59(5):891-9.

13. Kanwar VS, Sharma A, Srivastav AL, Rani L. Phytoremediation of toxic metals present in soil and water environment: a critical review. Environmental Science and Pollution Research. 2020 Sep 27:1-26. 